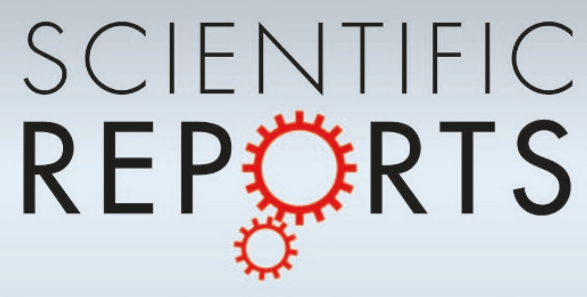

OPEN

SUBJECT AREAS:

CHEMICAL PHYSICS

SYNTHESIS AND PROCESSING

Received

29 May 2014

Accepted

28 July 2014

Published

14 August 2014

Correspondence and requests for materials should be addressed to Y.W. (wangyl@mail. ahnu.edu.cn) or M.L. (limaoguo@mail.ahnu. edu.cn)

\section{Selecting water-alcohol mixed solvent for synthesis of polydopamine nano-spheres using solubility parameter}

\author{
Xiaoli Jiang, Yinling Wang \& Maoguo Li
}

The Key Laboratory of Functional Molecular Solids, Ministry of Education, Anhui Key Laboratory of Chemo-Biosensing, College of Chemistry and Materials Science, Anhui Normal University, Wuhu 241000, People's Republic of China.

The solvent plays an important role in a given chemical reaction. Since most reaction in nature occur in the mixed-solvent systems, a comprehensive principle for solvent optimization was required. By calculating the Hansen solubility parameters (HSP) distance $R_{a}$, we designed a model experiment to explore the influence of mixed solvents on the chemical synthesis. The synthesis of polydopamine (PDA) in the water-alcohol system was chosen as model. As predicted, the well-dispersed PDA spheres were obtained in selected solvents with smaller $R_{\mathrm{a}}$ values: methanol/water, ethanol/water and 2-propanol/water. In addition, the mixed solvent with smaller $R_{a}$ values gave a higher conversion of dopamine. The strategy for mixed solvent selection is might be useful to choose optimal reaction media for efficient chemical synthesis.

T he solvent, which makes up approx. 90\% of the total mass of a reaction solution, plays an important role for a solution based chemical reaction. Solubility, equilibrium position and reaction rate are all affected by solvent ${ }^{1}$. Solvent selection continues to be one of the most challenging issues for current synthetic chemistry since most of chemical or biochemical reactions on the earth occur in wet environment ${ }^{2}$. Based on the principle of "like dissolves like", the single solvent system is the primary choice for chemical synthesis. However, most, if not all, reactions in nature occur in mixed-solvent systems. Up to now, the mixed solvent system has been widely used for many years, especially in the coating industry ${ }^{3}$. For example, using solvent selection by computer programs, one can now easily predict how to dissolve a given polymer in a mixture of two solvents, neither of which can dissolve the polymer by itself. Though mixed solvents have been used in synthetic chemistry, few investigations have focused on how to select mixed solvents.

Water and ethanol are versatile solvents, and the water-ethanol system has been broadly used. For example, mixtures with different fraction of ethanol have been used as alcoholic beverages. Another interesting example is that using 70 percent alcohol is a better disinfectant against the tubercle bacillus than using pure alcohol ${ }^{4}$. Recently, Zhang et al. developed a mixed-solvent strategy for efficient exfoliation of inorganic graphene analogues (IGAs), e.g., $\mathrm{MoS}_{2}, \mathrm{WS}_{2}$ and $\mathrm{BN}^{5}$. In this article, the authors demonstrated how two "poor" solvents, ethanol and water, can be designed to give high solubility to various IGAs.

Messersmith and co-workers demonstrated that dopamine self-polymerization was a powerful approach to apply multifunctional coatings onto various surfaces, including noble metals, metal oxides, ceramics, and polymers. These coatings would be served as an adhesion layer to immobilize biological molecules ${ }^{6}$. Consequently, several PDA-based systems have emerged as powerful tools to address many various challenging surface pro$\mathrm{blems}^{7-12}$. More recently, the monodisperse polydopamine (PDA) spheres have been directly synthesized in a water-alcohol mixed solvent at room temperature. The results revealed that the volume ratio of water to alcohol strongly influenced on the synthesis of the PDA spheres ${ }^{13}$. Lu and co-workers prepared PDA spheres by use of about $29 \%$ ethanol as the solvent ${ }^{14}$. To our knowledge, there was a lack of a comprehensive principle that applied to select the volume ratio of water to alcohol for PDA synthesis. Inspired by the above results, we therefore designed the experiments to explore the influence of solvent on the synthesis of PDA spheres in the water-ethanol system.

The theory of Hansen solubility parameters (HSP) is a semi-empirical correlation developed to explain dissolution behaviour ${ }^{15}$. Briefly, HSP formula usually has the symbols $\delta_{\mathrm{D}}, \delta_{\mathrm{P}}$, and $\delta_{\mathrm{H}}$, which are the dispersive, polar, and hydrogen-bonding solubility parameters, respectively, and are sometimes replaced by $D, P$, and $H$, for convenience. The dissolution process is an adaptation between the HSP parameters of solvents and solutes. The HSP distance $R_{\mathrm{a}}$ is used to evaluate the level of adaptation and $R_{\mathrm{a}}$ can be calculated by equation (1). 


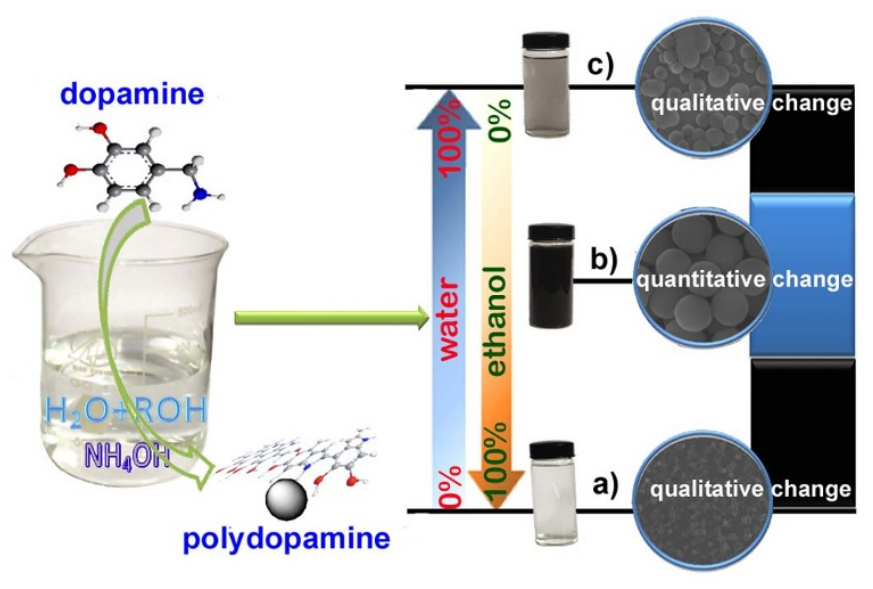

Figure 1 Synthesis of polydopamine in the water-alcohol mixed solvents. Photograph and SEM images of polydopamine in (a) pure ethanol, (b) the ethanol-water mixed solvent with the volume ratio of $30 \%$, and (c) pure water, respectively.

$$
R_{a}=\left[4\left(D_{\text {solv }}-D_{\text {solu }}\right)^{2}+\left(P_{\text {solv }}-P_{\text {solu }}\right)^{2}+\left(H_{\text {solv }}-H_{\text {solu }}\right)^{2}\right]^{0.5}
$$

The smaller the $R_{\mathrm{a}}$ value, the higher the expected solubility. In addition to single-component solvents, HSP theory can be also applied to mixed-solvents, in which each of the three HSP parameters for a solvent mixture is a linear function of composition, calculated by equation (2).

$$
D(P, H)_{b l e n d}=\sum \phi_{n, \text { comp }} D(P, H)_{n, c o m p}
$$

where $\phi$ is the volume fraction for each composition. Therefore, equation (1) and (2) enable us to predict the solubility of different chemicals in various mixed-solvents, which effectively allows us to design ideal solvent systems.

Before conducting experiments for the synthesis of PDA, $R_{\mathrm{a}}$ values between different solvent mixtures and dopamine were calculated using equation (1) and (2). More details are given in the Supporting Information (see Supplementary Materials online).

\section{Results \& discussion}

Figure 1 shows the results of experiments performed in the waterethanol mixed solvents. PDA spheres could not be formed in pure ethanol (Figure 1a), but the PDA spheres could be obtained in the mixed-solvents made with water and ethanol or pure water (Figure $1 \mathrm{~b}$ and 1c). Monodisperse PDA spheres could be synthesized only at an appropriate ratio of ethanol/water.

To verify the influence of solvent, we conducted the experiments to synthesize PDA in various water-ethanol mixed solvents. Figure 2 shows that the synthesis of PDA is strongly dependent on the volume fraction of ethanol in water. As shown in Figure 2c-f, the welldispersed PDA spheres were obtained in the mixed solvents with the volume fractions of ethanol from $25 \%$ to $40 \%$. We calculated the $R_{\mathrm{a}}$ between dopamine and ethanol/water mixtures using equations (1) and (2) based on the data listed in Table S1. Interestingly, these mixed solvents all have smaller $R_{\mathrm{a}}$ value (Table $\mathrm{S} 2$ ). In contrast, it was difficult to obtain well-dispersed PDA spheres in those mixed solvents with high $R_{\mathrm{a}}$ values. We also investigated the conversion of dopamine in the mixed solvents and found that a smaller $R_{\mathrm{a}}$ value gave a higher conversion (Table S5). Together, it seemed that the well-dispersed products with higher conversions tend to be prepared in the "good" solvents for the reactants.

It was worth mentioning that the concentration of ammonia had a dramatic effect on controlling the size of PDA spheres, but no effect on the morphology and yield of PDA sphere (Figure 3), consistent with previous reports ${ }^{14}$.

This alcohol-water system was not only limited to ethanol, other alcohols such as methanol or 2-propanol with smaller $R_{\mathrm{a}}$ were both proven to be efficient solvent systems. The $R_{\mathrm{a}}$ values between dopamine and methanol (or 2-propanol)/water mixtures are listed in Table S3 and S4. As predicted, the well-dispersed PDA spheres were obtained in $10 \%$ or $20 \%$ of methanol or $40 \% 2$-propanol with smaller $R_{\mathrm{a}}$ (Figure 4). Based on the analysis of the data, it was found that in all the three mixed solvent system the solvents with the smallest $R_{\mathrm{a}}$ gave the highest percent conversion of dopamine $(\rho)$, as shown in Tables S5-S7. It should be pointed that the $\rho$ was calculated via the weight ratio of PDA to DA and the morphology was ignored. Under optimum experiment conditions the $\rho$ in methanol, ethanol and
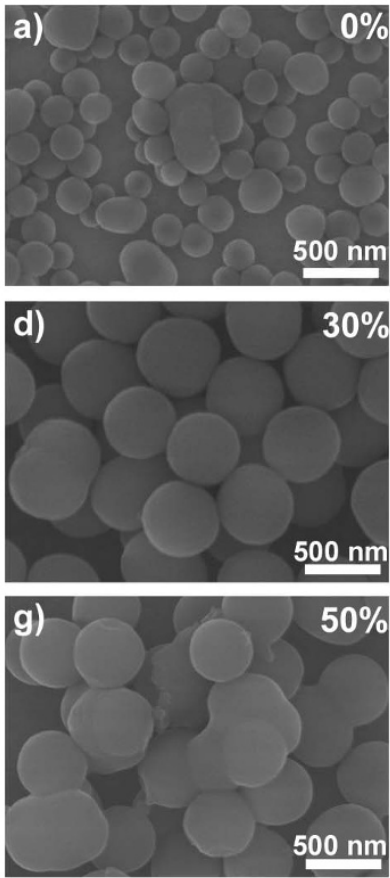
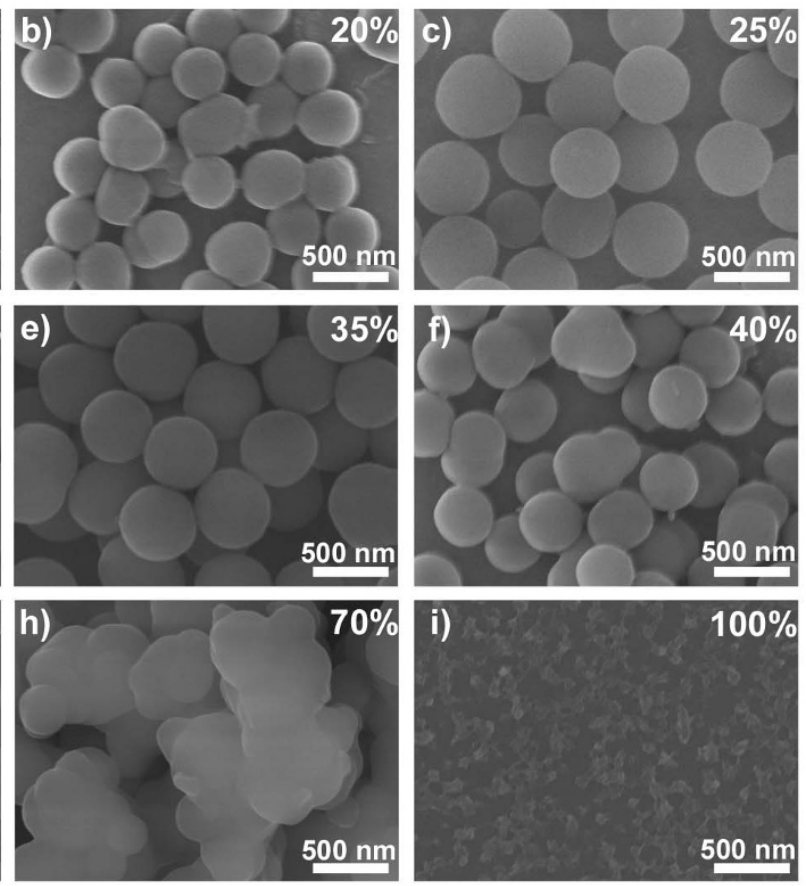

Figure 2 SEM images of PDA obtained in various ethanol/water mixtures. The volume fractions of ethanol are (a) $0 \%$, (b) $20 \%$, (c) 25 , (d) $30 \%$, (e) $35 \%$, (f) $40 \%$, (g) $50 \%$, (h) $70 \%$, and (i) $100 \%$, respectively. The scale bars are $500 \mathrm{~nm}$. 

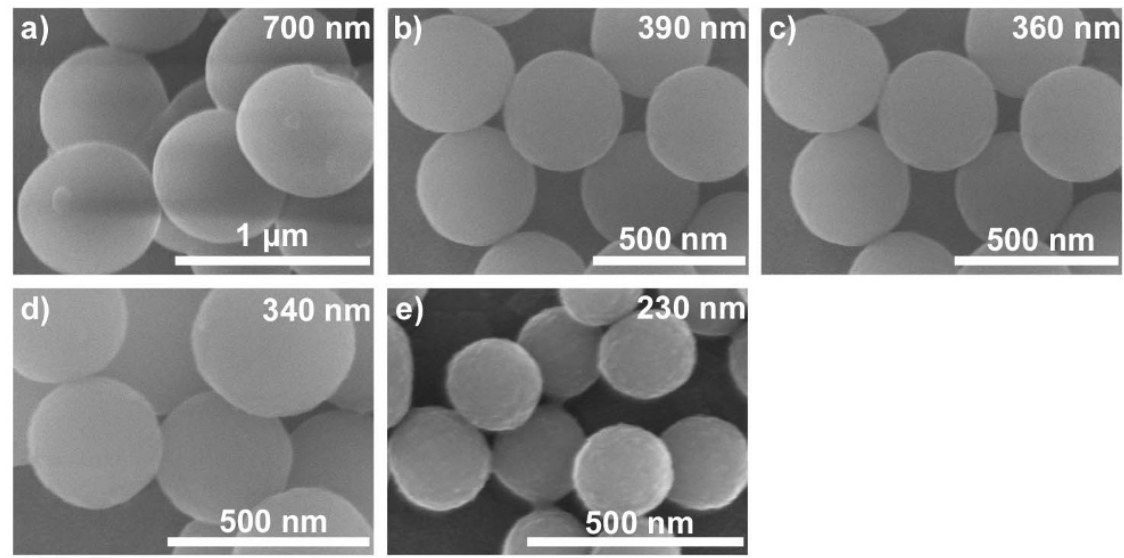

Figure 3 SEM images of PDA spheres with different diameters prepared at different concentration of ammonia, (a) 0.1 , (b) 0.17 , (c) 0.2 , (d) 0.28 , and (e) $0.35 \mathrm{~mL}(28-30 \%)$. The volume fractions of ethanol are all $30 \%$.

2-propanol-water system with smallest $R_{\mathrm{a}}$ was $50.57 \%, 40.25 \%$, and $37.18 \%$, respectively (Table S8). The yield of PDA spheres in methanol-water mixed solvent could even reach more than $50 \%$, indicating that the organic component of the mixed solvents is the main influence factor for the conversion of dopamine when using different solvents. Figure S1 and Figure S2 show the SEM images of PDA prepared in different methanol/water and 2-propanol/water mixtures. The PDA products tended to be assembled together in the solvents with higher $R_{\mathrm{a}}$.

The theory of chemical equilibrium may facilitate the understanding of this concept. The concentrations of the reactants or products do not affect the equilibrium constant, but does affect the equilibrium position ${ }^{16}$. For example, a decrease in concentration of the products causes the equilibrium to shift to the side of product. In other words, if the solvent can dissolve the reactants better than the products, the equilibrium will also tend to generate products. Apparently, the solubility of solvent is very important for chemical synthesis.

In summary, we demonstrated that the PDA spheres could be more easily obtained in the weakly alkaline alcohol-water mixed solvents with smaller $R_{a}$. Although the synthesis of PDA spheres in alcohol-water mixed solvents has been reported earlier ${ }^{13,14}$, the present report is the first example of the application of HSP theory for the prediction of the PDA synthesis in alcohol-water mixed solvents. This empirical formula might be useful to predict other reaction
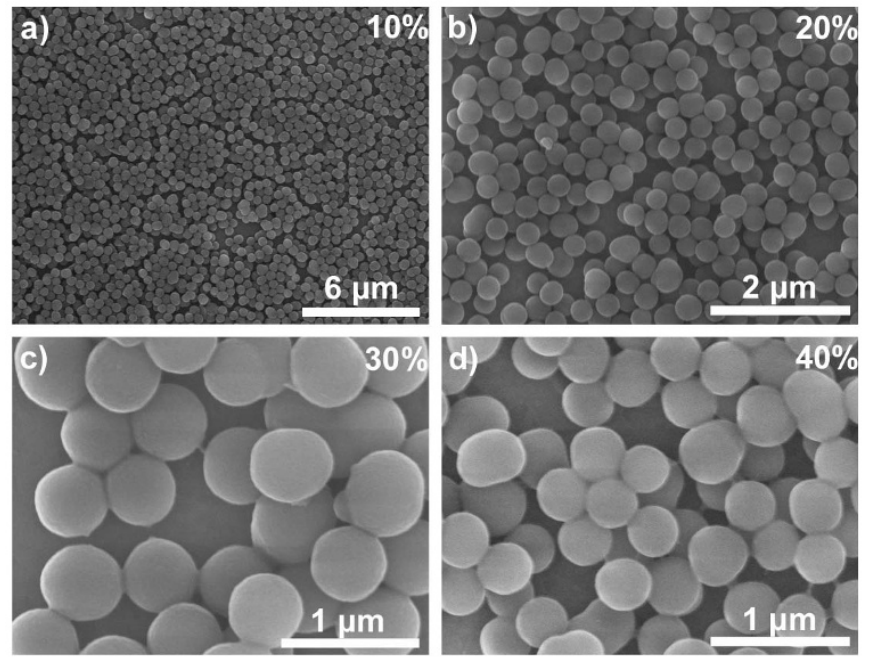

Figure 4 | SEM images of PDA obtained in (a), (b) methanol/water and (c), (d) 2-propanol/water mixtures. media in chemical synthesis. Further, with the aid of computers, more complex solvents with smaller $R_{\mathrm{a}}$ for reactants can be designed for synthesis. We expect to extend the selection of mixed solvents to other synthesis in inorganic, organic and biologic chemistry.

\section{Methods}

Materials. Dopamine hydrochloride (DA) was purchased from Acros Organics (New Jersey, USA). Ammonia aqueous solution, methanol, ethanol, and 2-propanol were obtained from J\&K Scientific Ltd (Shanghai, China). Other chemical reagents were of analytical grade and were obtained commercially. All the solutions were prepared with Milli-Q purified water (Millipore, $\geq 18.2 \mathrm{M} \Omega \mathrm{cm}$ ).

Preparation of PDA nano-spheres. The synthesis of PDA spheres was carried out in a water-alcohol mixed solvent. The alcohol (methanol, ethanol, or 2-propanol) was mixed with water and the volume of the mixed solvent was fixed at $40.0 \mathrm{~mL}$

Ammonia aqueous solution $\left(\mathrm{NH}_{4} \mathrm{OH}, 0.20 \mathrm{~mL}, 28-30 \%\right)$ was added to the alcohol/ water mixtures under mild stirring at room temperature for $30 \mathrm{~min}$. Finally, dopamine hydrochloride was directly added to the mixed solution. The color of this solution immediately turned to pale brown and gradually changed to dark brown. The reaction was allowed to proceed for $30 \mathrm{~h}$ according to the literature $\mathrm{e}^{14}$. The product was centrifuged and washed with water and anhydrous ethanol for three times. The product was dried in vacuum overnight at $60^{\circ} \mathrm{C}$ was used to calculate the conversion of dopamine.

Characterization. Field-emission SEM (Hitachi S4800) was used study the morphology of the products.

1. Buncel, E., Stairs, R. A. \& Wilson, H. The Role of the Solvent in Chemical Reactions (Oxford University Press, New York, 2003).

2. Chastrette, M., Rajzmann, M. \& Chanon, M. Approach to a general classification of solvents using a multivariate statistical treatment of quantitative solvent parameters. J. Am. Chem. Soc. 107, 1-11 (1985).

3. Hansen, C. M. Hansen Solubility Parameters: A User's Handbook (CRC Press, Hoboken, 2007).

4. Smith, C. R. Alcohol as a disinfectant against the tubercle bacillus. Public Health Rep. 62, 1285-1295 (1947).

5. Zhou, K. G. et al. A mixed-solvent strategy for efficient exfoliation of inorganic graphene analogues. Angew. Chem. Int. Ed. 50, 10839-10842 (2011).

6. Lee, H., Dellatore, S. M., Miller, W. M. \& Messersmith, P. B. Mussel-inspired surface chemistry for multifunctional coatings. Science 318, 426-430 (2007).

7. Wang, Y. L. et al. Multifunctional carbon nanotubes for direct electrochemistry of glucose oxidase and glucose bioassay. Biosens. Bioelectron. 30, 107-111 (2011).

8. Song, Q. et al. Bifunctional polydopamine@ $\mathrm{Fe}_{3} \mathrm{O}_{4}$ core-shell nanoparticles for electrochemical determination of lead(II) and cadmium(II). Anal. Chim. Acta 787, 64-70 (2013).

9. Zhang, L. et al. Combination of bioinspiration: a general route to superhydrophobic particles. J. Am. Chem. Soc. 134, 9879-9881 (2012).

10. Liu, Y., Ai, K. \& Lu, L. Polydopamine and its derivative materials: synthesis and promising applications in energy, environmental, and biomedical fields. Chem. Rev. 114, 5057-5115 (2014).

11. Sedó, J., Saiz-Poseu, J., Busqué, F. \& Ruiz-Molina, D. Catechol-based biomimetic functional materials. Adv. Mater. 25, 653-701 (2013).

12. Ye, Q., Zhou, F. \& Liu, W. Bioinspired catecholic chemistry for surface modification. Chem. Soc. Rev. 40, 4244-4258 (2011).

13. Yan, J. et al. Polydopamine spheres as active templates for convenient synthesis of various nanostructures. Small 9, 569-603 (2013). 
14. Ai, K. et al. $\mathrm{Sp}^{2} \mathrm{C}$-cominant $\mathrm{N}$-doped carbon sub-micrometer spheres with a tunable size: A versatile platform for highly efficient oxygen-reduction catalysts. Adv. Mater. 25, 998-1003 (2013).

15. Hansen, C. M. Hansen Solubility Parameters: A User's Handbook. (CRC Press, Hoboken, 1999).

16. Atkins, P. W. \& de Paula, J. Physical Chemistry. (Wiley-VCH, Weinheim, 2006).

\section{Acknowledgments}

We thank the National Natural Science Foundation of China (21075001) for support of this research. W.Y. also thanks the Anhui Provincial Natural Science Foundation (1408085QB27) for support.

\section{Author contributions}

X.J. performed the most of experiments, collected and analyzed the data. Y.W. wrote the main manuscript text and prepared all figures in text. M.L. conceived the experiments, analyzed results, and wrote the paper.

\section{Additional information}

Supplementary information accompanies this paper at http://www.nature.com/ scientificreports

Competing financial interests: The authors declare no competing financial interests.

How to cite this article: Jiang, X., Wang, Y. \& Li, M. Selecting water-alcohol mixed solvent for synthesis of polydopamine nano-spheres using solubility parameter. Sci. Rep. 4, 6070; DOI:10.1038/srep06070 (2014).

(c) (1) (2) This work is licensed under a Creative Commons Attribution-NonCommercialShareAlike 4.0 International License. The images or other third party material in this article are included in the article's Creative Commons license, unless indicated otherwise in the credit line; if the material is not included under the Creative Commons license, users will need to obtain permission from the license holder in order to reproduce the material. To view a copy of this license, visit http:// creativecommons.org/licenses/by-nc-sa/4.0/ 


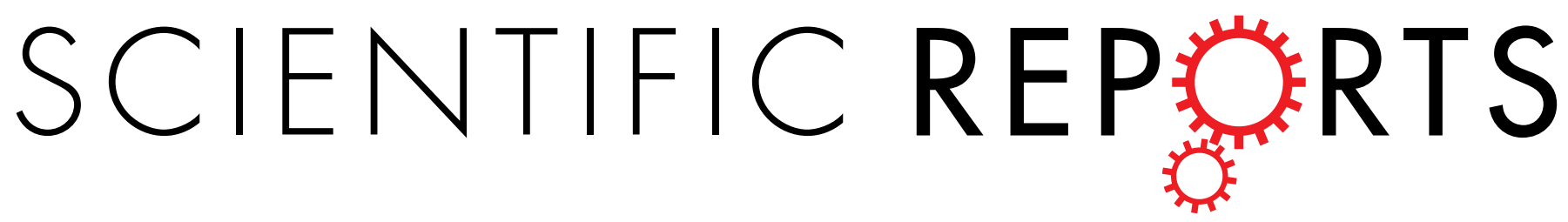

OPEN Corrigendum: Selecting wateralcohol mixed solvent for synthesis
of polydopamine nano-spheres
using solubility parameter

Xiaoli Jiang, Yinling Wang \& Maoguo Li

Scientific Reports 4:6070; doi: 10.1038/srep06070; published online 14 August 2014; updated on 01 September 2015

This Article contains errors in Figs 2 and 3. Figure 2d was inadvertently duplicated in Fig. 2e; Fig. 3c was inadvertently duplicated in Fig. 3b. The correct Figs 2 and 3 appear below as Figs 1 and 2 respectively.
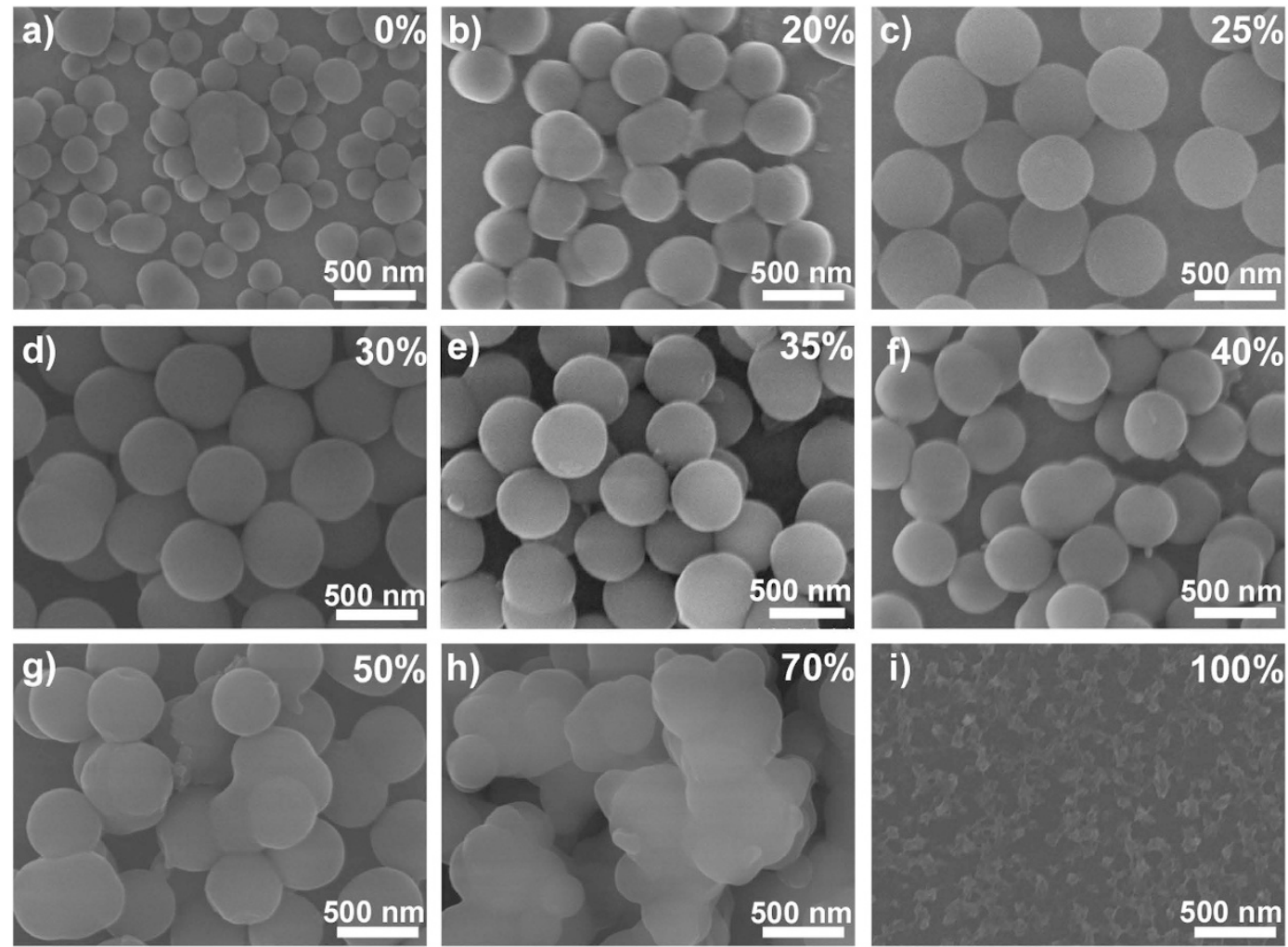

Figure 1. 

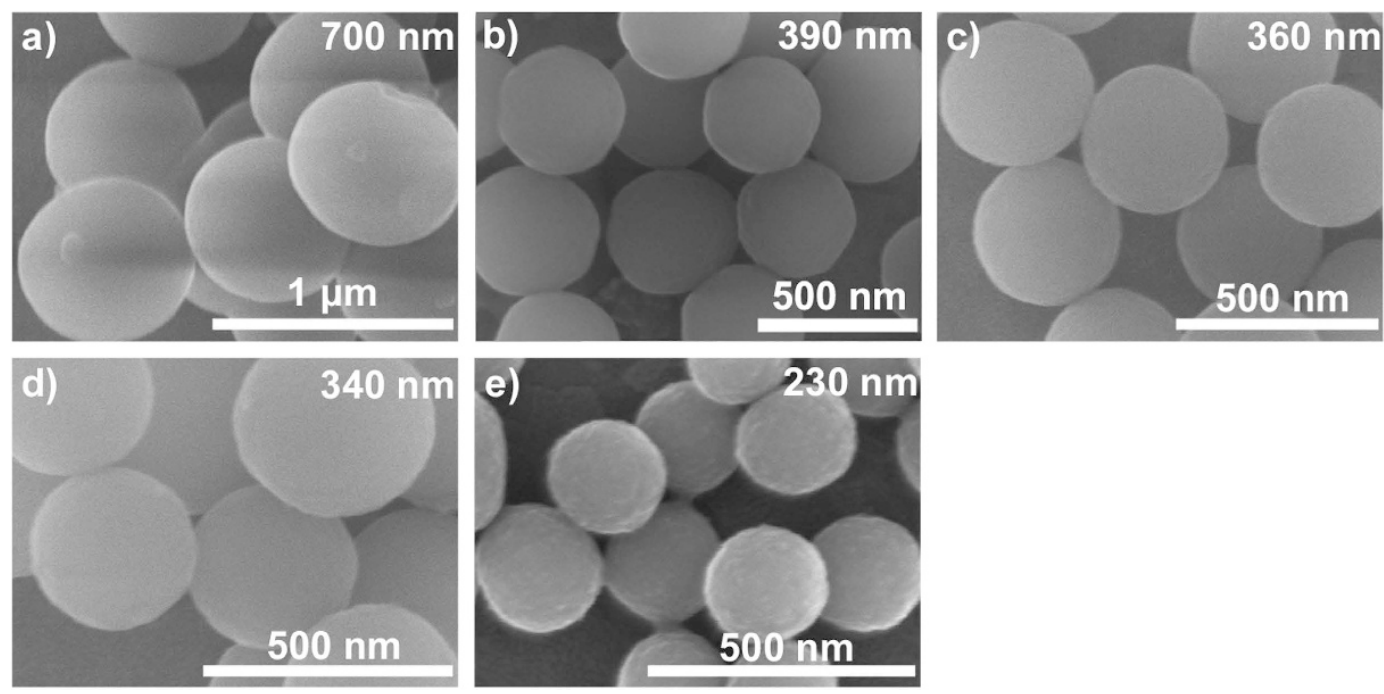

Figure 2 .

(i) This work is licensed under a Creative Commons Attribution 4.0 International License. The images or other third party material in this article are included in the article's Creative Commons license, unless indicated otherwise in the credit line; if the material is not included under the Creative Commons license, users will need to obtain permission from the license holder to reproduce the material. To view a copy of this license, visit http://creativecommons.org/licenses/by/4.0/ 